\title{
EFFICIENCY OF THE PREDICTION OF HIGH PRIORITY TRAFFIC IN ENHANCING THE RATE BASED CONTROL OF LOW PRIORITY TRAFFIC
}

\author{
Kimmo Pulakka and Jarmo Harju \\ Tampere University of Technology. Telecommunications Laboratory. P.O. Box 553 FIN-33101 \\ Tampere. FINLAND tel. +358-3-365 2111. fax +358-3-365 3808 \\ e-mail: (pulakka,harju)@cs.tut.fi
}

Key words: Flow control, traffic prediction, packet switched networks, fuzzy logic

Abstract: In this paper, we study how effectively the rate based control of a low priority data transmission service in a packet switched backbone network can be implemented, when the control decisions are based on the predictions of the amount of high priority traffic. ANFIS (adaptive neuro-fuzzy inference systems) predictors are used for traffic prediction. In our service model, all control functions of the low priority data transmission service are distributed to the edge switches of the backbone network. The routes and data rates of the low priority data flows are iteratively controlled by the control system, according to the predicted data rate variations of the high priority data flows. The efficiency of the service model has been tested by simulations. The emphasis is on the effects of different traffic distributions of the high priority data flows and on the impact and importance of the amount of overhead caused by the traffic prediction and the transmission of control information.

\section{INTRODUCTION}

In the Internet, a packet switched backbone network of one operator transfers data between access networks with end-users' computers and backbone networks of other operators. Data transmission services of future backbone networks can be roughly classified to the prioritised, controllable and best effort transmission services. The edge switches of the backbone

The original version of this chapter was revised: The copyright line was incorrect. This has been corrected. The Erratum to this chapter is available at DOI: 10.1007/978-0-387-35522-1_37 
networks classify the incoming data flows into different service categories, and the core switches of the networks just transfer the data flows through the networks according to the service category classifications [1]. The classification is mainly based on the negotiated service level agreements of the data flows.

The three above-mentioned service categories have their own individual characteristics. The data flows of the prioritised services are served by giving privileges over the other traffic in the network. The prioritised services can also include guaranteed services. For such services, the network guarantees a certain quality of the transmission services for these traffic flows. The controllable and best effort traffic can use the free capacity of the network, left over by the prioritised data flows. The data flows of the controllable service categories are always somehow controlled by the network so that the data flow spesific or general control targets are reached. Traditionally, the best effort traffic is not controlled in any way in the network level.

In the transmission environment where the different data flows should be served differently and the RTT (Round Trip Time) of the control actions may be very long, some kind of traffic predictions have proved to be useful in order to reach the near-optimal utilisation of the capacity of the network [2]. From this point of view, the main target of the prediction is to try to reduce the bad effects of large RTT of the control actions. These predictions can be used for example in CAC (Call Admission Control), UPC (Usage Parameter Control), traffic shaping, routing and data rate control functions. A common prediction strategy is to predict the behaviour of certain type of traffic flows so that some other type of traffic could be controlled optimally.

Traffic prediction can be made by using several prediction methods. However, a common characteristic for almost all prediction methods is to use the time series for the prediction. The next value or values of the certain characteristic of the traffic flows are predicted using the measured past characteristics of the traffic flows. During the past few years, several studies have been published where the behaviour of the VBR (Variable Bit Rate) type of traffic has been predicted using LMS (Least Mean Square), RLS (Recursive Least Square) or fuzzy logic based methods (e.g. [3] [4] [5] [6] ).

It is very difficult, if not impossible [7], to predict real data traffic in the network. As concluded in [7], the exact statistical characteristics of the predicted traffic should be known to produce good predictions. In real network environment, it is even impossible to collect the data fast enough to produce the exact statistical model of the predicted traffic. Hence, it is very important to think, how much computation power of the network components is used for different prediction tasks. In some situations, valuable predictions can be made using simple prediction algorithms. 
However, in the huge backbone networks with numerous different transmission services highly automated management tools are needed to control the network at least nearly optimally [8]. To implement these automated management tools properly, prediction algorithms with effective computational intelligence will be needed.

In this paper, we describe our own data rate and multipath route selection protocol for the low priority controllable (LPC) traffic, based purely on the traffic load predictions of the prioritised traffic. The main idea is to develop a low priority data transmission service, which does not require complex control functions all around the network, but which still would ensure better transmission service than the standard best effort data transmission service. The free capacity of the network, left over by the data flows of the prioritised data transmission services, is predicted and shared between the LPC data flows. All prediction functions are located at the edge switches of the network, and the core switches only collect the data rate information for the prediction tasks of the edge switches. From the network operator's point of view, this kind of service would use the free capacity of the network more effectively than the best effort service. On the other hand, the network applications, which traditionally use the best effort transmission service, would get faster transmission service. However, the network would not guarantee anything and the LPC sources would not be forced to behave exactly according to the control of the backbone network.

The main question is, what kind of prediction system is effective enough to be implemented instead of traditional best effort service. We use several performance parameters to study how effectively the LPC data transmission service could be implemented. Especially, we study the effect of the frequency of the predictions and the control operations with various traffic models of the prioritised traffic. The adaptive neuro-fuzzy inference system (ANFIS) [9] has been used as the prediction engine. The reason for this selection was that ANFIS has been found to be effective in modelling the behaviour of highly non-linear dynamic systems [10].

The developed data transmission service model is tested by the simulations. In these simulations, the prioritised data traffic was generated by three different models: 1) the first-order autoregressive process [11], 2) the measured sample data rates of the H.261 video transmissions, and 3) the fractional gaussian noise self-similar process with strictly defined value of the Hurst parameter. The simulated backbone network model does not follow any existing standardised network architecture. However, the modelled backbone network contains the typical elements of connection oriented high speed packet switched networks.

In Section 2 we explain the basic principles of our data transmission service, concentrating on the prediction operations of the high priority 
traffic. In Section 3 simulation results are given and analysed. Finally, some conclusions are drawn.

\section{DESCRIPTION OF THE CONTROL SYSTEM}

\subsection{Basic principles}

The studied backbone network model consists of edge and core switches. The access networks are connected to the backbone network via edge switches. The data packets coming from the source access networks flow across the source edge switches and travel through the core switches to the destination edge switches. The destination edge switches send the data packets to the destination access network (see Fig. 1). In this study, we expect that access networks are also some kind of packet based data networks, and it is possible to transfer special control information, related to our data transmission service, between the LPC source stations and edge switches of the backbone network. In the backbone network, data is transferred using constant length packets (cells). In the switches, every input and output link, connected to the switches, is handled by individual input and output ports. Output ports have a scheduler for the control traffic, prioritised traffic and LPC traffic. The control traffic has the highest priority and the LPC traffic has the lowest priority (see Fig. 2). Traditional best effort traffic is not included here, but if it were, its priority would be the lowest. In this study, we do not consider how the prioritised data flows are controlled. We just assume that their priority is always higher than the priority of the LPC data flows and their data rates are variable.

Source access network with source user stations

Source edge switch with control functions of LPC service

Core switches of the backbone network

Destination edge switch

Destination access network with destination user stations

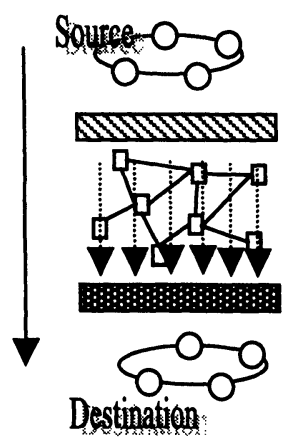

Figure 1. The data flow through the backbone network 
At the beginning of every LPC data transmission, the LPC source stations use certain nominal data rates, and the data packets of these data flows are transferred through the network using certain nominal routes between the source and destination edge switches. After that, the control system of the LPC transmission service iteratively adjusts the routes and the data rates of the active LPC data flows based on the predicted loads of the prioritised data flows. After every control moment, the edge switches inform the LPC source stations about the predicted optimal data rates and the corresponding optimal routes by using a special control protocol. Then it is the responsibility of the data transmission applications of the source stations to adjust their data rates according to received control information. Between two control moments, the LPC source stations send data using the optimal data rates of previously selected optimal routes, and the edge switches take care that the incoming data packets of the LPC data flows are sent through the backbone network using the previously defined optimal routes.

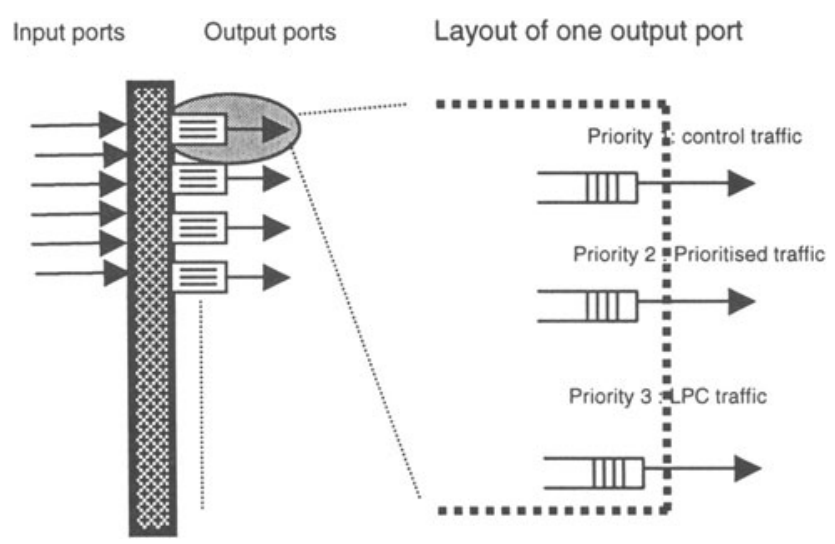

Figure 2. Layout of the core switches

The definitions of the optimal routes and optimal data rates of the LPC data flows are based on the predictions of the ANFIS predictors. For these predictions, the core switches of the backbone network measure the total data rate of the data flows of the prioritised transmission services in the output ports of the switches. To collect this information and to distribute it to all edge switches, the edge switches send periodically ERM (Edge Resource Management) packets to the network using a constant time interval $t_{s}$. These packets are flooded through the core switches to the other edge switches of the network, and the total data rate values of the prioritised traffic and the number of the active LPC flows in the most heavily loaded output ports of 
the switches in each route are copied to the ERM packet. After receiving any ERM packet, the edge switch copies the control information of the ERM packet to its special data base.

At a constant time interval $t_{c}$, the ANFIS predictor systems of the edge switches copy the latest control information from the special data base for the prediction operations. The ANFIS predictions are made in the constant time interval $t_{p}\left(t_{p}=n^{*} t_{c}, n \in Z_{4}\right)$. In this way, the ANFIS predictors collect the set of the past data rate values of the prioritised data flows, calculate the values of the input variables of the ANFIS predictors using these values, and predict the maximum data rate values of the prioritised traffic flows in the near future. The prediction time is equal to $t_{p}$ and the predictions are done individually for every route between the edge switches. At the end of the predictions, the optimal routes and data rates for the LPC sources are calculated, and the control operations described above are done. The control loop of the LPC data transmission service is described in Fig. 3.

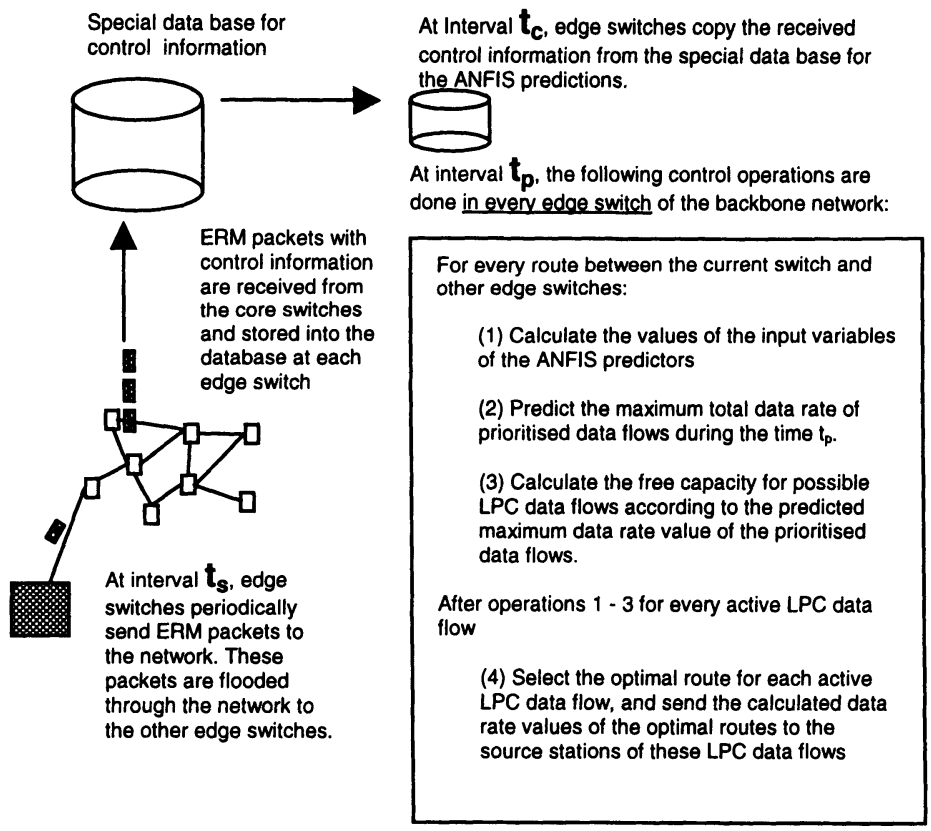

Figure 3. Control operations of the LPC data transmission service

It is important to notice that the values of the interval parameters $t_{s}, t_{c}$ and $t_{p}$ define how strictly the LPC data flows are controlled. If short intervals are used, the data rates of the prioritised data flows can be predicted more accurately, and the data rates of the LPC sources are controlled nearly 
optimally. The price to pay is the huge amount of the control operations of the network components. On the other hand, using long intervals reduces the computaional as well as control traffic overhead, but at the same time the accuracy of the control operations can be lost. We assume that the performance of the ANFIS predictors decrease when the prediction time increase. Finally, the operators of the backbone networks should decide how much resources of the network components is profitable to use to control the LPC data flows, and set the values of the interval parameters according to this decision. In Section 3, we will study how the values of the interval parameters affect the performance of the LPC data transmission service and how much network capacity is used in each case.

Before the ANFIS predictors can be used, their parameters must be set for the current prediction case. The parameters are always set in the off-line mode. At first, the statistically representative sample values of the data rates of the prioritised data flows are collected in the routes between the edge switches. After that, the input-output data pairs are constructed and the parameters of the ANFIS predictors are set by the special hybrid learning algorithm [9] of the ANFIS network. As a result, the ANFIS predictors can predict the data rates of the prioritised data flows in the near future. It is important to notice that the exactness of the ANFIS predictions depends strongly on the 'goodness' of the sample data rates used for learning. The performance of the ANFIS predictions decrease, if they should do the predictions for the cases, which are statistically far from the prediction cases used for the parameters setting processes. In Section 3, we will also study how exact predictions the ANFIS predictors calculate in the cases where statistical characteristics of the data rates differ from the statistical characteristics of the data rates used for the parameter setting process of the ANFIS predictor.

\subsection{Description of the ANFIS predictors}

The ANFIS network is an adaptive network-based fuzzy inference system, which can perform the functions of the Sugeno or Tsukamoto fuzzy models. The important characteristic of the network is the capability to learn the behaviour of the dynamic systems by training data pairs. The network includes a set of linear and non-linear parameters. The values of the parameters are adjusted by a special hybrid learning rule, which is a combination of the gradient method and the least squares estimate (LSE). The ANFIS network can include several input variables but only one output variable [9].

In our predictions case, three input variables have been chosen to describe the dynamic characteristics of the prioritised data flows. These three 
variables are 1) the maximum of the data rate values of the prioritised data flows during the measurement time $t_{p}, 2$ ) the mean of the data rate values of the prioritised data flows during the measurement time $t_{p}$, and 3) the difference between the last two data rate measurements of the prioritised data flows during the measurement time $t_{p}$. The output value of the ANFIS predictor is the maximum predicted total data rate of the prioritised data flows in the currently predicted route.

The configuration of the ANFIS network affects to the performance of the ANFIS predictions. The values of several factors used during the parameter setting process and the number of the membership functions of the input variables can be changed. For example, the increase of the number of the membership functions usually allows more exact prediction results. However, this also requires more sample data pairs and computation time to set the parameters of the ANFIS predictors properly. Finally, the configuration of the ANFIS predictors should be selected according to the characteristics of the predicted processes. The selections can be made by the experience of the system manager or by the special algorithms like CART (classification and regression tree) [13]. In section 3, we will compare the prediction results of two ANFIS predictors with different number of the membership functions of the input variables.

\subsection{Selection of optimal routes and definition of optimal data rates}

At the end of each control moment of the LPC data transmission service, the control system selects the optimal routes for the active LPC data flows and defines the allowed data rates for these flows. The route selections depend on 1) the current and predicted loads of the prioritised and LPC data flows, and the current load of the control traffic in the routes, 2) the number of active LPC data flows in the routes, and 3) the randomised orders of the route selections of different $L P C$ data flows.

At first, the order in which the optimal routes between the LPC data flows are selected is randomised. After this, the optimal routes for LPC data flows are selected in the randomised order.

During every route selection, the route with the largest equally shared free capacity is defined as an optimal route and the current value of the equally shared free capacity is defined as an optimal data rate of the LPC data flow. After the selection, the equally shared free capacity of the selected route must be decreased to ensure that the next route selections of other LPC data flows are based on the right information. 


$$
\mathrm{EQS} \text { capacity }=\frac{\text { Link capacity }-(\mathrm{CT}+\text { Pred.PRIO }+ \text { Curr.LPC }+ \text { Pred.LPCC })}{\# \text { of the active GC traffic flows }}
$$

Where :

$$
\begin{array}{ll}
\text { EQS capacity } & =\text { Equally shared free capacity } \\
\text { CT } & =\text { Current control traffic } \\
\text { Pred.PRIO } & =\text { Predicted load of prioritised traffic } \\
\text { Curr.LPC } & =\text { Load of current LPC traffic } \\
\text { Pred.LPCC } & =\text { Predicted load changes of LPC traffic }
\end{array}
$$

\section{SIMULATIONS}

\subsection{Description of the simulated network environment}

The simulated network environment consisted of one backbone network and three access networks. The capacity of the transmission links between the switches of the backbone network was $35 \mathrm{Mbit} / \mathrm{s}$, and the transmission capacity of the access networks was $100 \mathrm{Mbit} / \mathrm{s}$. The high transmission capacities of the access networks ensure that no congestion can occur in the access networks in any case.

The backbone network was loaded by five continuously flowing prioritised traffic flows and four LPC data flows with randomly alternating idle and active periods. Poisson and exponential distributions were used to define the lengths of the active and idle periods of the LPC data flows. The feasible mean lengths of the active periods were 5, 10 and 20 seconds, and the mean lengths of the idle periods were 4, 8 and 12 seconds. Transmission rates of five prioritised data flows were generated by 1) the first-order autoregressive process, 2) measured sample data rates of the H.261 video transmissions, or 3) the fractional gaussian noise self-similar process. The value of the Hurst parameter in the self-similar process was 0.9 .

Data packets of all data flows were of equal length. The constant data packet size was 500 bytes. The optimal lengths of the LPC buffers and the distances between the switches of the network were also constant. The buffer size was 7000 packets and the distance between the switches was $500 \mathrm{~km}$. The control system tried to share the free capacity between the LPC traffic flows so that the network would have been loaded optimally. Another control target was to prevent congestion in the network. The structure of the simulated network environment is described in Fig. 4. 
The aim of the test series was to 1) measure the 'goodness' of the ANFIS predictor system compared to two other predictor systems, and 2) to study the effects of different values of the interval parameters $t_{s}, t_{c}$ and $t_{p}$ to the performance of the LPC data transmission service. The test series includes seven test cases. The test cases differ from each other by the used prediction methods and the data rate distributions of the prioritised traffic flows. Each test case includes four different test phases. The test phases differ from each others by the value sets of the interval parameters $t_{s}, t_{c}$ and $t_{p}$. The simulation time of each test phase was 250 seconds.

The same value sets of the interval parameters and traffic scenarios of the LPC data flows were used for the test phases of the all test cases. Hence, it is possible to compare the performance results of different test cases. The characteristics of the simulation tests are summarised in Table 1.

In the tests, the distributions used to set the parameters of the ANFIS predictors were different from the distributions used to measure the performance of the ANFIS predictors. In this way, we tried to study what would be the realistic prediction capability of the ANFIS predictors. The data rate distributions of the prioritised data flows, measured in the output ports from the edge switch one to the core switches two, three and seven, are described in Figure 5. The data rate generation processes of the prioritised data flows are described in Table 1.

Table 1. Description of the tests

\begin{tabular}{ll}
\hline Issue & Characteristics \\
\hline data-rate generation process 1 & Aim: Generate data rates of prioritised data flows to \\
measure the performance of the ANFIS predictors. \\
Data rate generator: fractional gaussian noise self- \\
similar process. The value of the Hurst parameter \\
was 0.9. \\
Mean and std values, measured in the output \\
ports between the edge switch 1 and core switches \\
2,3, and 7: mean= 5.76, std = 2.67 \\
Aim: Generate data rates of prioritised data flows to \\
measure the performance of the ANFIS predictors. \\
Data rate generator: First-order autoregressive \\
process together with measured data rates of H.261 \\
video transmissions. \\
Mean and std values, measured in the output \\
ports between the edge switch 1 and core switches \\
$\mathbf{2 , 3}$ and 7: mean=3.43, std = 1.55. \\
Aim: Generate data rates of prioritised data flows to \\
set the parameters of the ANFIS predictors. \\
Data rate generator: fractional gaussian noise self- \\
similar process. The value of the Hurst parameter \\
was 0.9. \\
Mean and std values, measured in the output \\
\hline
\end{tabular}




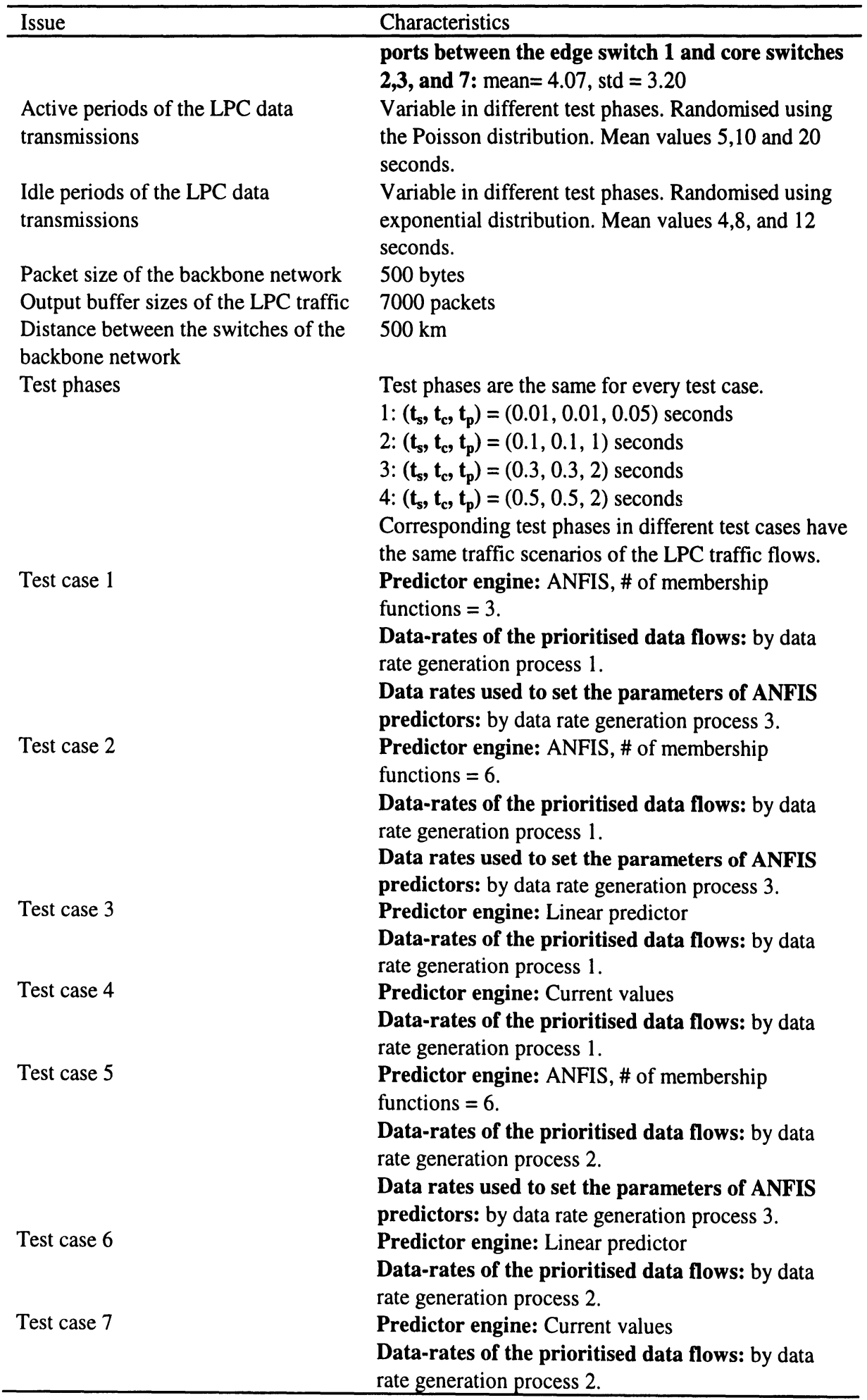




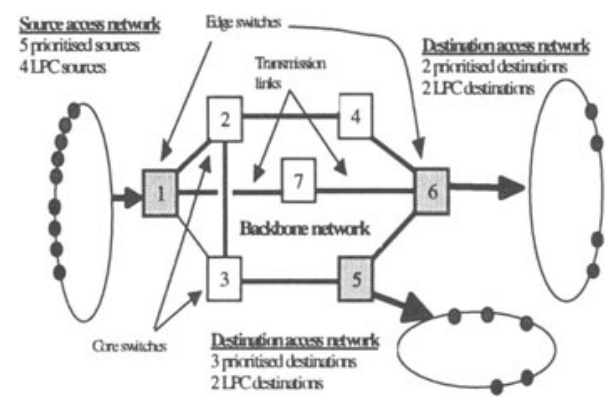

Figure 4. Simulated network environment

Data rate distributions of the prioritised traffic

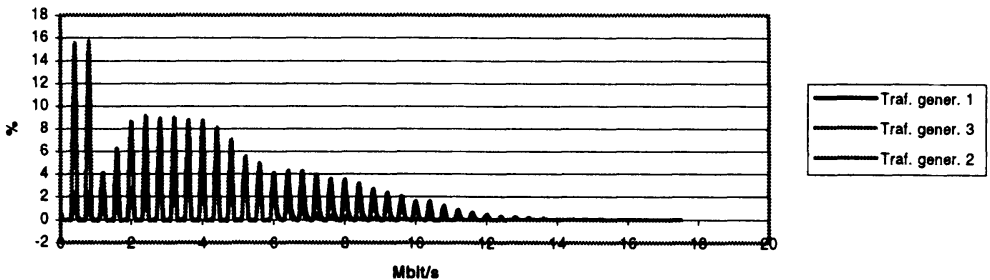

Figure 5. Alternative data rate distributions of the prioritised traffic flows

\subsection{Performance variables}

The performance of the LPC transmission service is tested by five performance variables. These variables are: 1) the average occupancy of the LPC buffers in the three most heavily loaded output ports, 2) the standard deviation of the occupancy of these LPC buffers, 3) the packet loss ratio, 4) mean throughput, and 5) the average and the standard deviation of the prediction error of the data rate values of the prioritised data flows. The values of the mean throughput were obtained by calculating, for each LPC data flow, the ratio of the achieved throughput to the theoretical throughput value given by the max-min fairness principle [12] under the assumption that $100 \%$ of the available capacity of the network was used. The prediction errors were measured between the predicted and measured data rates of the prioritised data flows.

The combination of the high throughput values and zero packet loss indicate the high performance of the system. The values of the average occupancy levels and standard deviations of the LPC buffers indicate how the buffers of the output ports are used. In our simulated network 
environment, we have measured the performance values for the output buffers from the switch one to the switches two, three and seven. These output ports should always be used heavily, because all incoming LPC traffic injected to the backbone network flows through the switch one. The single output port is used optimally, if the average value of the occupancy level of the LPC buffer is between zero and the optimal size of the LPC buffer, and the value of the standard deviation is low. In our control system, the low values of the standard deviation cannot be reached. The control system adaptively changes the routes of the LPC traffic flows, and this causes quite high variation of the occupancy levels in the output buffers of the switch one.

On the other hand, the small values of the interval parameters $t_{s}, t_{c}$ and $t_{p}$ require high overhead for the control of LPC data flows. In these tests, the prediction error values should be lower and throughput values should be higher than in the tests with large values of the interval parameters.

\subsection{Description and analysis of the test results}

At first, we will concentrate on the test case 1 (see Table 1). We will study how the different value sets of the interval parameters $t_{s}, t_{c}$ and $t_{p}$, used in four separate test phases, affect to the performance results. Secondly, we will measure the performance differences of seven test cases. According to these test results, we will study how valuable the ANFIS predictors are to implement LPC data transmission service.

The test results of the test case 1 are summarised in Table 2. According to the test result, the mean throughput decreases when the values of the interval parameters increase. However, the difference between the throughput results of the best and worst tests is only $19 \%$, although the values of the interval parameters vary heavily. At same time, the difference between the prediction errors and the packet loss ratios of different tests is small. Obviously, the average and standard deviation values of the LPC buffers increase, when the values of the interval parameters increase. In these cases, the LPC sources are controlled slower and the buffer occupancy levels can vary heavily, before the control operations of the LPC data transmission service affect to the occupancies of the LPC buffers.

The performance results of seven test cases are summarised in Table 3. The values of the performance variables in Table 3 are the average performance results of four different test phases (see Table 1) of the test cases. 
Table 2. Effects of the values of the interval parameters

\begin{tabular}{lllll}
\hline $\begin{array}{l}\text { Test phases (1-4) } \\
\left(\mathrm{t}_{\mathrm{s}}, \mathrm{t}_{\mathrm{c}}, \mathrm{t}_{\mathrm{p}}\right)\end{array}$ & $\begin{array}{l}\text { Mean } \\
\text { throughput }\end{array}$ & Packet loss ratio & $\begin{array}{l}\text { Prediction error } \\
\text { (mean, std) } \\
\text { [ Mbit/s ] }\end{array}$ & $\begin{array}{l}\text { Occupancy of } \\
\text { LPC buffers } \\
\text { (mean, std) } \\
\text { [Packets ] }\end{array}$ \\
\hline $0.01,0.01,0.05$ & 0.92 & 0.0009 & $1.61,1.68$ & 162,795 \\
$0.1,0.1,1$ & 0.78 & 0.0022 & $1.58,1.63$ & 482,1258 \\
$0.3,0.3,2$ & 0.77 & 0.0068 & $1.73,1.74$ & 511,1418 \\
$0.5,0.5,2$ & 0.75 & 0.0074 & $2.07,2.00$ & 569,1470 \\
\hline
\end{tabular}

According to the test results of test cases 1-4, we could conclude that the LPC data transmission service implemented by the well constructed ANFIS predictor produces better transmission service than the other alternatives. The performance results of the test case 1 are generally better than the performance results of any other test case. However, the throughput value in test case 2 is same as in test case 1, although the ANFIS predictor used in test case 2 includes more membership functions of the input variables than the ANFIS predictor used in test case 1 does. At the same time, the prediction error values of test case 1 are clearly better than in the error values of test case 2 . This result indicates that more sample data pairs are needed to set the parameters of the ANFIS when the number of parameters in ANFIS increases. In these two cases, exactly same data pairs were used to set the parameters of the ANFIS predictors of both test cases. The ANFIS predictor in test case 2 would need more data pairs and calculation iterations of the hybrid leaning method to set the parameters of the ANFIS predictor to reach the optimal prediction results.

Although the performance results of test cases 3 and 4 are worse than the result of test case 1 , the performance differences are small. It is important to notice that the performance results of test cases 3 and 4 are reached by clearly less computations than needed for ANFIS predictor, even if the ANFIS predictor would not include huge number of parameters.

In test cases 5-7, the data rates of the prioritised data flows were generated by the non self-similar process (Table 1 , data rate generation process 2 ). In these test cases, the data rates should be easier to predict than the data rates used for test cases 1-4. The average and standard deviation values of the prediction errors in these cases are smaller than the error values in test cases 1-4. However, the performance differences between ANFIS prediction based data transmission service (test case 5) and the other services, based on simpler prediction methods, are smaller than those measured between test cases 1-4. The prediction error values of ANFIS predictor are even larger than the error values of simpler prediction methods. In this point of view, the value of the ANFIS predictors decreases compared to the simpler prediction methods when the data rates of the prioritised data flows are easier to predict. 
In the case of the prediction of the maximum data rate values of the prioritised data flows, the optimal prediction results of the data rates do not ensure the optimal throughput results. In some load situations, the good throughput and packet loss results can be reached even if the prediction error values are large.

Table 3. The performance results of different test cases

\begin{tabular}{|c|c|c|c|c|}
\hline $\begin{array}{l}\text { Test cases } \\
(1-7)\end{array}$ & $\begin{array}{l}\text { Mean } \\
\text { throughput }\end{array}$ & $\begin{array}{l}\text { Packet } \\
\text { loss ratio }\end{array}$ & $\begin{array}{l}\text { Prediction error } \\
\text { (mean, std) } \\
{[\mathrm{Mbit} / \mathrm{s}]}\end{array}$ & $\begin{array}{l}\text { Occupancy of } \\
\text { LPC buffers } \\
\text { (mean, std) } \\
\text { [ Packets ] }\end{array}$ \\
\hline $\begin{array}{l}\text { (1) anf3, self-similar } \\
\text { prior. traffic }\end{array}$ & 0.81 & 0.0043 & $1.75,1.76$ & 431,1235 \\
\hline $\begin{array}{l}\text { (2) anf6, self-similar prio. } \\
\text { traffic }\end{array}$ & 0.81 & 0.0049 & $2.38,2.24$ & 441,1227 \\
\hline $\begin{array}{l}\text { (3) Linear predictor, self- } \\
\text { similar prio. traffic }\end{array}$ & 0.75 & 0.0058 & $2.14,1.96$ & 329,961 \\
\hline $\begin{array}{l}\text { (4) Current values, self- } \\
\text { similar prio. traffic }\end{array}$ & 0.74 & 0.0029 & $2.02,1.93$ & 262,831 \\
\hline $\begin{array}{l}\text { (5) anf6, autoreg. and } \\
\text { H. } 261 \text { based prio. traffic }\end{array}$ & 0.78 & 0.0064 & $1.77,1.82$ & 415,1257 \\
\hline $\begin{array}{l}\text { (6) Linear predictor, } \\
\text { autoreg. and H. } 261 \text { based } \\
\text { prio. traffic }\end{array}$ & 0.74 & 0.0061 & $1.38,1.23$ & 123,631 \\
\hline $\begin{array}{l}\text { (7) Current values, } \\
\text { autoreg. and H.261 based } \\
\text { prio. traffic }\end{array}$ & 0.72 & 0.0038 & $1.25,1.17$ & 310,943 \\
\hline
\end{tabular}

\section{CONCLUSIONS}

In this paper, the low priority data transmission service is described. The transmission service is based on the iterative route selections and data rate adjustments of the sources. The control operations of the transmission service are based purely on the data rate predictions of the prioritised data flows. The predictions are done by the ANFIS predictors.

The performance of the transmission service is tested by the simulations. In these simulations, the performance of the transmission service is measured as a function of several parameters. The simulation results indicate that the transmission service can adapt to different situations without heavy variations of the performance of the service. It is also found that ANFIS predictors perform better than the tested simpler predictors do. The ANFIS predictors are better especially in those prediction cases where the data rates of the prioritised data flows are difficult to predict. However, the test results show that the maximum throughput difference between the ANFIS predictor 
and tested simpler predictor systems is only $8.6 \%$. At same time, the implementation of the ANFIS predictors is a much more complex task than the implementation of the simpler prediction methods. The ANFIS predictors must also be configured carefully, before they produce good performance results. However, ANFIS predictors require much less computations than the prediction methods based on some other computationally intelligence algorithms (e.g. Multi-Layer Perception networks) [13].

\section{REFERENCES}

[1] Rosenberg, S.; Aissaoui, M.; Galway, K.; Giroux, N. , Functionality at the edge: designing scalable multiservice ATM networks, IEEE Communications Magazine, pages: 88 - 90, 95-9, May 1998, Vol. 36

[2] Ahmet Sekercioglu, Y.; Pitsillides A., Intelligent control techniques for efficient requlation of ABR queue length in ATM switches, Proceedings. Second IEEE Symposium on Computers and Communication (Cat. No. 97TB100137), p 80-84

[3] Randhawa, T.S.; Hardy, R.H.S. Estimation and prediction of VBR traffic in high-speed networks using LMS filters. International Conference on Communications, 1998. ICC 98. Pages: 253 - 258 vol.1

[4] Randhawa, T.S.; Hardy, R.H.S. Application of AR based model in proactive management of VBR traffic. 1998 1st IEEE International Conference on ATM. ICATM-98. Pages: 234 - 241

[5] Bin Qiu; Liren Zhang; Wu, H.R. Fuzzy multi-step ahead prediction of VBR video sources. 1997 International Conference on Information, Communications and Signal Processing, 1997. ICICS. Pages: $1623-1626$ vol. 3

[6] Qixiang Pang; Shiduan Cheng; Peng Zhang. Adaptive fuzzy traffic predictor and its applications in ATM networks. 1998 IEEE International Conference on Communications, 1998. ICC 98. Pages: 1759 1763 vol. 3

[7] Hall, J.; Mars, P. The limitations of artificial neural networks for traffic prediction. Third IEEE Symposium on Computers and Communications, 1998. ISCC '98. Pages: $8-12$.

[8] Callon, R. Predictions for the core of the network. IEEE Internet Computing. Jan.-Feb. 2000. Vol. 4. Pages: $60-61$.

[9] J.-S. R. Jang. ANFIS: Adaptive-Network-Based Fuzzy Inference System. IEEE Transactions on Systems, Man, and Cybernetics. Vol. 23 no. 3. May/June 1993. Issue 3, Pages: 665 - 685.

[10] J.-S.R. Jang, C.-T. Sun, Predicting chaotic time series with fuzzy if-then rules. Second IEEE International Conference on Fuzzy Systems 1993. Pages 1079-1084. vol 2.

[11] Mischa Schwartz. Broadband Integrated Networks. Prentice-Hall, A Simon \& Schuster Company, Upper Saddle River, New Jersey. Section 3.5

[12] D. Bertsekas, R. Gallager. Data Networks. Second edition. Prentice-Hall, Englewood Cliffs, New Jersey, 1992. Section 6.5.2.

[13] J.-S. R. Jang, C.-T. Sun, E. Mizutani. Neuro-Fuzzy and Soft Computing - A Computational Approach to Learning and Machine Intelligence, Prentice Hall, Upper Saddle River, NJ 07458. 